Article

\title{
The Platelet Fraction Is a Novel Reservoir to Detect Lyme Borrelia in Blood
}

\author{
Victoria P. Sanderson ${ }^{1}\left(\mathbb{D}\right.$, Iain L. Mainprize $\left.{ }^{1} \mathbb{(}\right)$, Lisette Verzijlenberg ${ }^{1}$, Cezar M. Khursigara ${ }^{2}(\mathbb{D}$ \\ and Melanie K. B. Wills ${ }^{1, *(\mathbb{D})}$ \\ 1 G. Magnotta Lyme Disease Research Lab, Molecular and Cellular Biology, University of Guelph, Guelph, \\ ON N1G 2W1, Canada; vsande01@uoguelph.ca (V.P.S.); imainpri@uoguelph.ca (I.L.M.); \\ lisette.verzijlenberg@gmail.com (L.V.) \\ 2 Molecular and Cellular Biology, University of Guelph, Guelph, ON N1G 2W1, Canada; \\ ckhursig@uoguelph.ca \\ * Correspondence: mwills@uoguelph.ca; Tel.: +1-519-824-4120 (ext. 54062); Fax: +1-519-837-1802
}

Received: 16 September 2020; Accepted: 27 October 2020; Published: 29 October 2020

check for

Simple Summary: To diagnose Lyme disease, a patient's blood is tested for antibodies that develop as part of the immune response. This can lead to cases being missed or inadequately treated. An ideal test would directly detect the Lyme disease bacteria, Borrelia, to provide better clinical guidance. In this study, we aimed to improve the methods currently used to find Borrelia in human blood, and identified two opportunities for optimization. We demonstrate that the container most commonly used to collect blood (EDTA) decreases Borrelia's ability to grow, and we identify a superior alternative (citrate). Additionally, using experimentally infected blood, we show that Borrelia is highly concentrated in the platelet fraction, making it an ideal candidate for direct detection. These results lay the foundation for diagnostic test development, which could improve patient outcomes in Lyme disease.

\begin{abstract}
Serological diagnosis of Lyme disease suffers from considerable limitations. Yet, the technique cannot currently be replaced by direct detection methods, such as bacterial culture or molecular analysis, due to their inadequate sensitivity. The low bacterial burden in vasculature and lack of consensus around blood-based isolation of the causative pathogen, Borrelia burgdorferi, are central to this challenge. We therefore addressed methodological optimization of Borrelia recovery from blood, first by analyzing existing protocols, and then by using experimentally infected human blood to identify the processing conditions and fractions that increase Borrelia yield. In this proof-of-concept study, we now report two opportunities to improve recovery and detection of Borrelia from clinical samples. To enhance pathogen viability and cultivability during whole blood collection, citrate anticoagulant is superior to more commonly used EDTA. Despite the widespread reliance on serum and plasma as analytes, we found that the platelet fraction of blood concentrates Borrelia, providing an enriched resource for direct pathogen detection by microscopy, laboratory culture, Western blot, and PCR. The potential for platelets to serve as a reservoir for Borrelia and its diagnostic targets may transform direct clinical detection of this pathogen.
\end{abstract}

Keywords: Lyme disease; Borrelia; blood processing; EDTA; citrate; platelets; molecular detection; OspA; culture; diagnosis

\section{Introduction}

Lyme disease, a tick-vectored zoonosis caused by a clade of bacteria within the Borrelia genus, is a growing public health threat that requires more rapid and sophisticated laboratory diagnostics to 
mitigate the escalating impact [1,2]. The early stage of the infection can present as non-specific flu-like symptoms as well as an erythema migrans (EM) rash, which was once considered pathognomonic of Lyme [3]. However, the utility of using the EM as a primary diagnostic feature has been challenged by findings of relatively low rash prevalence, variable and inconsistent manifestation [4], difficulty distinguishing the lesion on darker skin tones [5], and the reported appearance of non-borrelial EMs $[6,7]$. Prompt detection and antibiotic treatment of Lyme in the early stages of disease provides the best prognosis, although symptoms may reoccur and evolve in up to one third of patients [8,9]. Without adequate intervention, Borrelia burgdorferi sensu lato (s.l.) (henceforth B. burgdorferi, Borrelia, or $\mathrm{Bb}$ ) can disperse through vasculature and lymphatics to distal locations including the nervous system and heart, giving rise to serious multisystem manifestations that can resemble other diseases [10]. Such mimicry can further delay and complicate diagnosis.

The standard laboratory test for Lyme disease consists of two-tiered serological assessment of IgG and/or IgM response, which suffers both biological and technical limitations [11]. As an indirect test of infection, it relies on the adaptive host immune response, which can be slow to develop, is itself a target of the pathogen, and cannot be used to discern active infection from past exposure [10]. Serological testing is further complicated by microbiological diversity, intrinsic and extrinsic factors that blunt the immune response [12], low-throughput analysis, and inter-lab variability [13].

Alternatively, direct testing techniques such as clinical culture [14], DNA-based methods [15], and antigen detection [16] have long been investigated for their potential to report infection in a sensitive, specific, and timely manner, using accessible biospecimens. To date, however, their application to Lyme disease has been constrained by low and inconsistent spirochetemic burden, Borrelia preference for secondary tissue sites, limited understanding of host-adapted Borrelia characteristics, and a lack of standardized blood processing methods to recover limited pathogen material in circulation. Depending on the assay, technical challenges can also arise from the dilution effect of host materials, which may obscure the target(s) of interest.

Although blood is an attractive, minimally invasive analyte, estimates of Borrelia concentration vary considerably, and appear to depend on the stage of disease, pathogen genotype, pre-analytical methodology, and quantification technique. Vascular burden is anticipated to be highest during the initial dispersal from the tick bite site [17], yet most Lyme Borrelia cannot be found using microscopy of peripheral blood [18], and PCR-based DNA detection from serum or plasma during the first month of infection has an average estimated sensitivity range of only 34-62\% [19]. This may reflect strain-level differences in propensity for hematogenous dissemination [20], as well as methodological inconsistencies. Few studies have attempted to quantify the number of Borrelia per unit volume of blood, but among those that have, the resulting estimates span orders of magnitude. Early work suggested that there were, on average, fewer than 50 Borrelia genomes/mL of plasma [21], with a range of less than 20 spirochetes/mL to more than $4000 / \mathrm{mL}$ [22]. More recently, a study of early untreated Lyme patients detected a Borrelia chromosomal locus by quantitative PCR (qPCR) in $34 \%$ of acute patients; within those samples, 4660 copies of the gene were found on average per $\mathrm{mL}$ of plasma [23]. Meanwhile, culture-based analyses have estimated 0.1 cultivable organisms $/ \mathrm{ml}$ of blood in acute disease [24]. In 2016, a new member of the pathogenic Lyme Borrelia complex (B. mayonii) was discovered that presents with spirochetemia estimated to be 180 times higher than that of conventional B. burgdorferi s.l. [18], emphasizing interspecific variability and the clinical relevance of biodiversity. Overall, however, the consensus holds that bacterial load in blood is minor and transient in Lyme disease [2]. Advances in molecular detection technologies suggest that the low concentrations of colonizing Borrelia will be surmountable $[2,25,26]$, particularly if the recovery and analysis of the pathogen or its biomarkers can be further optimized.

Here, we used experimentally infected human blood to evaluate the impact of blood collection and processing on Borrelia availability and cultivability, and identified that the most common approaches can be detrimental to the organism and the sensitivity of downstream assays. This proof-of-concept work provides an experimental basis for the optimization of blood handling in diagnostic protocols 
to provide the greatest opportunity for Borrelia detection. These methodological improvements can now be applied to the analysis of samples from patients with acute and late disease to determine their impact on assay sensitivity.

\section{Materials and Methods}

\subsection{Comparison of Clinical Culture Protocols}

Studies were compiled by searching PubMed for (("Borrelia burgdorferi") AND ("culture")) AND ("blood") with the requirement that blood-based clinical culture was conducted in the study and both inoculation source and collection tube were clearly indicated. The search was conducted on 15 January 2020, and performed again on 23 June 2020 to capture recent publications. The data collected included the blood components that were used for inoculation of culture, the anticoagulant present in collection tubes for whole-blood and plasma collection, and whether centrifugation steps were implemented for serum and plasma separation from whole blood.

\subsection{Bacteria}

Reference strain Borrelia burgdorferi s.s. B31 (ATCC 35210) (referred to as B. burgdorferi, Borrelia, $\mathrm{Bb}$, and $\mathrm{B} 31$ throughout the manuscript) was used to conduct all experiments, except the culture of Borrelia from isolated blood fractions where a GFP Borrelia strain was used (obtained through Juan Salazar, University of Connecticut). Bacterial stocks were stored at $-80^{\circ} \mathrm{C}$ in BSK with $20 \%$ glycerol. Cultures were propagated at $37^{\circ} \mathrm{C}, 5 \% \mathrm{CO}_{2}$ in BSK-H medium with $6 \%$ rabbit serum (BB83-500, Dalynn Biologicals, Calgary, AB, Canada; referred to as BSK throughout manuscript). Bacterial culture was always counted using Petroff-Hausser counting chambers under phase-contrast light microscopy.

\subsection{Vacutainer Anticoaguant Cell Viability Assessments}

Ethylenediaminetetraacetic acid (EDTA) exposure was modelled using a $6 \mathrm{~mL}$ Becton Dickinson (BD) Biosciences vacutainer K2 EDTA (K2E) $10.8 \mathrm{mg}$ blood collection tube (BD 368661, Becton, Dickinson and Company, Franklin Lakes, NJ, USA). Citrate exposure was modelled using $4.5 \mathrm{~mL}$ BD buffered sodium citrate (9NC) $0.105 \mathrm{M}=3.2 \%$ blood collection tube (BD 369714, Becton, Dickinson and Company). Serum collection and controls were modelled using $10 \mathrm{~mL}$ uncoated BD serum blood collection tube clot activator vacutainer (BD 367820, Becton, Dickinson and Company). In each tube, $5 \%$ of the available volume was left unoccupied to provide a microaerophilic environment in accordance with B. burgdorferi B31 growth recommendations. The remaining volume was filled with BSK and B31 at a 9:1 ratio. Thus, for EDTA tubes, $0.57 \mathrm{~mL}$ B31 culture was added to $5.13 \mathrm{~mL}$ BSK. For citrate exposure, $0.428 \mathrm{~mL}$ B31 culture was inoculated into $3.85 \mathrm{~mL}$ BSK. In uncoated tubes, $0.95 \mathrm{~mL}$ B31 was seeded into $8.55 \mathrm{~mL}$ BSK or for non-growth controls, and $9.5 \mathrm{~mL}$ BSK was added to the uncoated tube. In each biological replicate, a 4-5-day old mid-log phase B31 culture was used to inoculate vacutainers. Vacutainers were loaded using needle and 3-cc syringes to mimic blood draw and maintain vacuum seal. Inoculated vacutainers were then incubated on ice in a Styrofoam container for $48 \mathrm{~h}$ to mimic shipping. Following this incubation, vacutainers were opened, and $1 \mathrm{~mL}$ was removed and seeded into $6.5 \mathrm{~mL}$ of fresh BSK in $8 \mathrm{~mL}$ polystyrene round-bottom tubes (Falcon 352027, Corning Life Sciences, Tewksbury, MA, USA). Freshly seeded cultures and vacutainers were then placed at $37^{\circ} \mathrm{C}$, $5 \% \mathrm{CO}_{2}$ and counted weekly by Petroff-Hausser counting chamber phase-contrast light microscopy (DHC-N01, INCYTO, Republic of Korea). This procedure was conducted in biological triplicate on three separate occasions with a fresh culture each time. Technical replicates were accounted for through Petroff-Hausser counting chamber of five separate squares.

\subsection{Experimentally Infected Blood Preparation}

All experiments involving blood samples were conducted under University of Guelph (UoG) Research Ethics Board (REB) approval number 18-07-007 (amendment approved 1 March 2019 ). 
After providing informed consent, healthy individuals with ties to the G. Magnotta Lyme Disease Research Lab donated blood for the purpose of protocol optimization and validation. All blood draws were performed by a qualified phlebotomy technician using previously approved venipuncture procedures (UoG SOP014) in the Human Nutraceutical Research Unit clinical trial suite at the University of Guelph. There were no official inclusion criteria for the research study and no fasting requirements. Blood was drawn into sodium citrate vacutainers (BD 369714, Becton, Dickinson and Company) for the purpose of whole-blood collection or uncoated vacutainers (BD 367815, Becton, Dickinson and Company) for serum collection. Blood samples were placed on ice and immediately transported to the lab for inoculation with B. burgdorferi B31. To test the impact of centrifugation, B31 was inoculated into whole blood at a 1:1 ratio of culture to whole blood, which resulted in an MOI of $1 \mathrm{Bb}: 137 \mathrm{RBC}$. For experiments involving microscopy and molecular analyses of the four blood fractions, $200 \mu \mathrm{L}$ of whole blood was inoculated with $2 \mathrm{~mL}$ BSK alone for uninfected controls or $2 \mathrm{~mL}$ of B31 culture in BSK to an MOI of $1 \mathrm{Bb}: 18.37 \mathrm{RBC}$. This dilution of blood was intended to improve visualization of blood cell-Borrelia interactions. For culture from blood fractions, $1 \mathrm{~mL}$ of whole blood was inoculated with $2 \mathrm{~mL}$ of Borrelia (MOI = $1 \mathrm{Bb}: 250 \mathrm{RBC}$ ). Experimentally infected serum was collected by allowing the spiked blood to clot and subsequently spinning the liquid at $1000 \times g$, for $10 \mathrm{~min}$, at $20^{\circ} \mathrm{C}$. Following inoculation of whole blood, experimentally infected and uninfected samples were always incubated for $30 \mathrm{~min}$ at $37^{\circ} \mathrm{C}$ with a $200 \mathrm{rpm}$ of rotation in a shaking incubator to prevent separation of whole blood before any further processing.

\subsection{Blood Fractionation by Centrifugation}

All centrifugation steps were conducted using a Sorvall Legend XTR centrifuge (Thermo Fisher Scientific, Burlington, ON, Canada) with a Thermo Tx-1000 75,003,017 rotor. Following the incubation step, a portion of infected and uninfected whole blood was set aside, while the remainder was fractionated by standard centrifugation protocols. In the initial experiment testing the direct impacts of centrifugation on stratification of Borrelia, one spin was conducted at $400 \times g$ and $20^{\circ} \mathrm{C}$, for $20 \mathrm{~min}$, to separate cell components (red blood cells and platelets) from liquid plasma. The cell component was diluted 1:1 with 1× PBS (phosphate-buffered saline) for ease of visualization, and this was accounted for in all calculations. Further experiments used two centrifugation steps, allowing the collection of whole blood (pre-centrifugation), a red blood cell-enriched fraction (first pellet), plasma, and a platelet-enriched fraction (second pellet). To separate red blood cells from platelet-rich plasma, whole blood was spun at $120 \times g$ and $20^{\circ} \mathrm{C}$, for $20 \mathrm{~min}$, from which infected and uninfected red blood cells were collected. Platelet-rich plasma was then separated using a second centrifugation step at $400 \times \mathrm{g}$ and $20^{\circ} \mathrm{C}$, for $20 \mathrm{~min}$, from which infected and uninfected plasma were collected, as well as infected and uninfected platelet pellets (resuspended in $100 \mu \mathrm{L}$ of PBS) [27]. A portion of each of the collected fractions was stored at $-20^{\circ} \mathrm{C}$ while the rest was used for additional analyses, as described below.

\subsection{Immunofluorescent Slide Preparation and Microscopy}

Matched infected and uninfected samples for whole blood, red blood cells, plasma and/or platelets were used to prepare slides. Slides were prepared by placing $4 \mu \mathrm{L}$ of the desired sample onto a microscope slide (16004-382, VWR International, Mississauga, ON, Canada) and smearing with a second slide. Slides were then allowed to air dry for a minimum of $5 \mathrm{~min}$ before fixation by dipping in $100 \%$ methanol for $30 \mathrm{~s}$. Slides prepared for the initial one-step centrifugation experiment were then mounted with Prolong Gold Antifade with DAPI (Cell Signaling Technology, Inc., Danvers, MA, USA) which contains 4',6-diamidino-2-phenylindole (DAPI) for DNA detection without any additional staining. These slides were imaged by phase-contrast light microscopy using the Leica DM2000LED microscope (Leica Microsystems, Concord, ON, Canada). Slides prepared for immunofluorescent staining were placed in $1 \times$ PBS overnight after methanol fixation and stained the following day. Immunofluorescent staining was conducted using the following steps: slides were blocked in 5\% BSA 
in PBS for one hour, washed twice in $1 \times$ PBS, incubated with primary antibody cocktail for $1 \mathrm{~h}$ (Platelet $\mathrm{IIb} / \mathrm{III}$ a mouse monoclonal at 1:50 (Santa Cruz Biotechnology, Inc., Dallas, TX, USA) and Acris1002 anti-Borrelia rabbit polyclonal at 1:500 in 5\% BSA(Origene Technologies, Inc., Rockville, MD, USA) followed by three $1 \times$ PBS washes, incubated with secondary antibody cocktail for $1 \mathrm{~h}$ (AlexaFluor 555 goat anti-mouse 1:500, AlexaFluor 488 donkey anti-rabbit 1:200 in 5\% BSA (A-21424 and A-11029, ThermoFisher Scientific, Burlington, ON, Canada)) and mounted with Prolong Gold Antifade with DAPI. Slides were imaged by phase-contrast light microscopy and fluorescence on Leica DM550B. Fluorescent and phase-contrast images were combined into composites using ImageJ. Three smears were conducted for each of the three biological replicates and three images were captured for each slide. Counting of total cells and interactions between cells was conducted using the ImageJ CellCounter plugin (https://imagej.nih.gov/ij/plugins/cell-counter.html) to produce average technical and biological replicates.

\subsection{Culture from Blood Fractions}

Experimentally infected platelet and serum fractions were collected as described above, and then $1 \mathrm{~mL}$ of the sample was inoculated into $6.5 \mathrm{~mL}$ BSK in $8 \mathrm{~mL}$ polystyrene round-bottom tubes (Falcon 352027). Cultures were incubated at $37^{\circ} \mathrm{C}, 5 \% \mathrm{CO}_{2}$, and counted weekly. Tubes were always inverted prior to counting.

\subsection{Western Blotting}

Experimentally infected and uninfected samples of each fraction were prepared by adding $16 \mu \mathrm{L}$ of the sample to $160 \mathrm{~mL}$ of lysis buffer $\left(10 \%\right.$ glycerol, $50 \mathrm{mM}$ Hepes pH 7.5, $150 \mathrm{mM} \mathrm{NaCl}, 1.5 \mathrm{mM} \mathrm{MgCl}_{2}$, $1 \mathrm{mM}$ EGTA, $10 \mathrm{mM} \mathrm{NaPPi}, 100 \mathrm{mM} \mathrm{NaF}, 1 \%$ Triton X-100 plus ProteaseArrest (786-108, G-BioSciences, St. Louis, MO, USA) and PhosphataseArrest (786-450, G-BioSciences) and then incubating at room temperature for $20 \mathrm{~min}$. Following lysis, samples were centrifuged at $12,000 \times \mathrm{g}$ and $4{ }^{\circ} \mathrm{C}$, for $5 \mathrm{~min}$, and the supernatant was frozen at $-20^{\circ} \mathrm{C}$, subsequently referred to as the fraction lysate. After, $5 \times \mathrm{SDS}$ loading buffer (250 mM Tris-HCl, pH 6.8, 10\% SDS, 30\% glycerol, 0.1\% Bromophenol Blue, 0.5\% beta-mercaptoethanol) was added to the fraction lysate at a 1:4 ratio. A volume of $20 \mu \mathrm{L}$ of the sample was then loaded into the wells of a 12.5\% SDS-PAGE gel. Electrophoresis was conducted using a BioRad Mini Protean Electrophoresis System Bio-Rad Laboratories (Canada) Ltd. (Mississauga, ON, Canada) with the following parameters: $50 \mathrm{~V}, 20 \mathrm{~min}$ then $150 \mathrm{~V}, 50 \mathrm{~min}$. The gel was then placed in transfer buffer ( $3 \mathrm{~g} / \mathrm{L}$ Tris base, $14.4 \mathrm{~g} / \mathrm{L}$ glycine, $\mathrm{dH}_{2} \mathrm{O}, 25 \%$ methanol) for $15 \mathrm{~min}$. A PVDF membrane (TM300-0.45PVDF, FroggaBio, Concord, ON, Canada) was activated in methanol and placed in water. Transfer was conducted on the Pierce ${ }^{\mathrm{TM}}$ Power Blotter (Thermo Fisher Scientific, Burlington, ON, Canada) with the following parameters: $0.8 \mathrm{~A}, 25 \mathrm{~V}, 7 \mathrm{~min}$. Following protein transfer, the membrane was blocked with $5 \%$ skim milk in TBS (Tris-buffered saline) overnight. The membrane was then washed briefly in TBS-T (TBS + 0.1\% Tween-20) then incubated with the primary antibody for $3 \mathrm{~h}$ (SC58093 OspA mouse monoclonal, Santa Cruz Biotechnology, Inc., Dallas, TX, USA). The membrane was washed 3 times in TBS-T for 10 min each. A volume of $2 \mu \mathrm{L}$ ECL anti-mouse IgF, HRP-linked secondary antibody (Cytiva, Global Life Sciences Solutions Canada ULC, Mississauga, ON, Canada) was added to $20 \mathrm{~mL}$ of $5 \%$ skim milk. Secondary antibody incubation was conducted for $1 \mathrm{~h}$. The membrane was washed in TBS-T for 10 min each. All original western blots can be found in Figure S1.

\subsection{Polymerase Chain Reaction (PCR)}

Each PCR reaction was prepared in $0.2 \mathrm{~mL}$ single PCR tubes with $16.4 \mu \mathrm{L}$ PCR water, $2.5 \mu \mathrm{L} 10 \times$ reaction buffer, $2 \mu \mathrm{L} 25 \mathrm{mM} \mathrm{MgCl} 2,0.5 \mu \mathrm{L}$ dNTPs, $0.1 \mu \mathrm{L}$ Taq DNA polymerase (ThermoFisher-EP0402 recombinant $5 \mathrm{U} / \mu \mathrm{L}), 1.25 \mu \mathrm{L} 10 \mu \mathrm{M}$ forward primer, $1.25 \mu \mathrm{L} 10 \mu \mathrm{M}$ reverse primer, and $1 \mu \mathrm{L}$ template. Primers amplified the variable region of the 16S rRNA gene. Primer sequences were as follows: Primer 1 ATGCACACTTGGTGTTAACTA and Primer 2 GACTTATCACCGGCAGTCTTA [28]. Template DNA was unprocessed (no DNA isolation) and directly added as the experimentally infected blood fraction 
and the PCR reaction was run with recommended conditions. Each amplified sample was then separated on a $1 \%$ ultra-pure agarose gel with loading buffer consisting of $6 \times$ DNA loading buffer ( $30 \%$ glycerol, $0.25 \%$ orange G dye). The gel was stained with $3 \times$ RedSafe for $30 \mathrm{~min}$, and imaged by UV illumination.

\subsection{Statistical Analyses}

All statistical analyses were conducted using Prism8 (GraphPad Software, San Diego, CA, USA).

\section{Results}

\subsection{Appraisal of Existing Clinical Culture Practices}

Although the recovery of Borrelia from patient samples has routinely been attempted for several decades, no standardized protocol exists. We therefore set out to curate the LD direct detection literature to evaluate blood collection and processing methods. Specifically, we sought to identify the range of approaches used and the strength of evidence upon which these methodological choices are based, and to determine whether the field is converging on a consensus strategy. The stepwise process of drawing blood for direct testing begins with venipuncture and blood collection into a vacutainer, which is either uncoated (yielding serum), or treated with one of EDTA, sodium citrate, or heparin anticoagulants, from which plasma and various cell fractions can be obtained. A systematic review of NCBI-indexed literature relating to B. burgdorferi blood culture allowed for compilation of inoculum sources, anticoagulants, and centrifugation steps for subsequent analysis. These results are summarized in Figure 1 and Table A1, and reveal that Borrelia clinical culture protocols have varied widely $[9,14,22,29-46]$. Blood or skin biopsy from EM rash are the most common inoculum sources, and although the latter is informative in a subset of early infection cases, it is largely not applicable to ongoing disease. Within the cohort of studies that inoculate from blood, there is also a range of blood components used, with serum (9/28 relevant experiments) and plasma (13/28 relevant experiments) being the most common. These 28 experiments correspond to the 21 independent studies presented in Table A1, as several studies use multiple parallel inoculum sources. Culture from plasma reportedly leads to a greater chance of recovery compared to serum [29,34,42,47]. A small number of studies have incorporated cellular components or whole blood $[9,22,42]$. The most common anticoagulant vacutainer used for whole-blood and plasma collection is EDTA (11/28 relevant experiments) followed by citrate (3/28 relevant experiments), and although several studies used heparin in the 1990s (5/28 relevant experiments), this trend has not continued. Although one relatively recent investigation, which scored Borrelia recovery from a mixture of blood from spirochetemic SCID mice and healthy humans, suggested that EDTA was not ideal for this purpose [48], the anticoagulant remains in use. Once blood has been collected, some studies allow for natural clotting (serum) or separation (plasma), while others centrifuge blood to obtain the desired component $[29,34]$. At least $60 \%$ of studies implemented centrifugation with speeds ranging from $100 \times g$ to $1100 \times g$ for the purpose of either serum or plasma separation. Following inoculation, cultures are typically monitored at a particular interval using dark-field microscopy and then confirmed using some combination of molecular techniques such as PCR (with or without sequencing), immunostaining, and high-resolution microscopy. The time frame within which cultures are monitored varies significantly, ranging from 4 to 16 weeks, with or without subcultivation. 


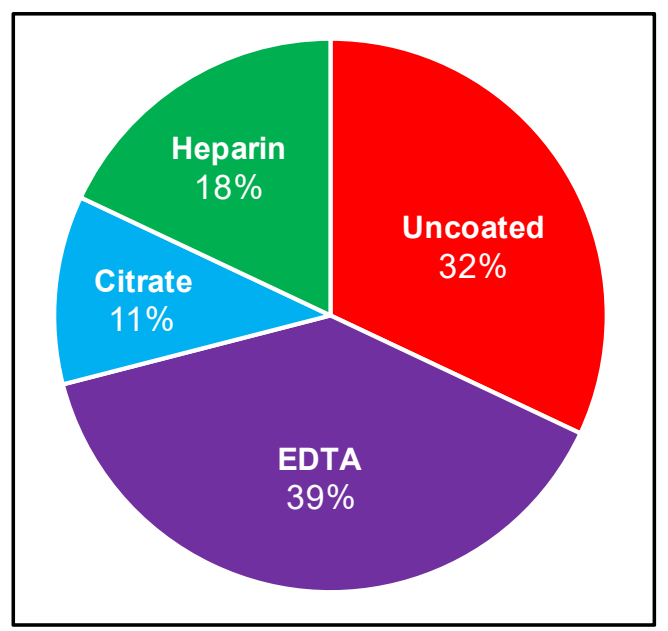

Figure 1. Reported use of anticoagulants to recover Borrelia from human blood. Relevant literature was curated (as described in Materials and Methods) to compare which vacutainers are commonly used when conducting Borrelia clinical culture from patient samples. Percentage calculated as number of times the vacutainer was used relative to the total experiments conducted using vacutainers in a set of applicable studies.

Overall, there is no consensus approach to Borrelia recovery, and the procedural differences have made it challenging to compare studies, draw conclusions about optimal techniques for clinical detection, or reconcile incongruent results in Lyme disease patient samples [2]. The most common approaches include the use of plasma, collected using EDTA, and processed with centrifugation, although robust evidence supporting these choices is lacking. It was therefore of interest to evaluate the effectiveness of common approaches, and provide an experimental basis for the standardization and optimization of blood handling for Borrelia detection.

\subsection{Effect of Anticoagulants on Borrelia Viability}

Of the three anticoagulants that have been used for Borrelia recovery, heparin was eliminated from our experimental design at the outset due to previously documented contraindications. It is a known inhibitor of molecular analyses, particularly PCR, and is also a well-characterized ligand of Borrelia [49,50], which could further confound observations. Perhaps as a consequence, heparin has fallen out of use in Lyme disease investigations since the late 1990s (see Table A1). There are precedents in the literature to suggest that all anticoagulants used for recovery of whole blood may impact growth to a certain degree, but the relative inhibition of growth at the microbiological level has not yet been assessed [48]. In this experiment, B. burgdorferi strain B31 cultured in liquid BSK medium was seeded directly in the EDTA, citrate, and uncoated vacutainers, and either grown in that environment or subcultured in fresh broth and counted weekly using a Petroff-Hausser chamber.

Uncoated/unaltered culture reached peak growth by 7 days, followed by the stationary/death stage. Citrate- and EDTA-exposed cultures followed similar growth trends, but were unable to reach the same maximum concentration of cells. After 7 days of growth directly in the vacutainer environment, Borrelia cultures in citrate tubes achieved a higher cell concentration than those in EDTA ( $p=0.0103)$ (Figure 2A). Similarly, after subculture, Borrelia inoculated from citrate vacutainers recovered to within range of the unaltered cultures, reaching $85 \%$ of the positive control growth $(p=0.4934)$ while Borrelia exposed to EDTA only reached $53 \%$ of the uncoated positive control $(p=0.0221)$ (Figure 2B). 
A

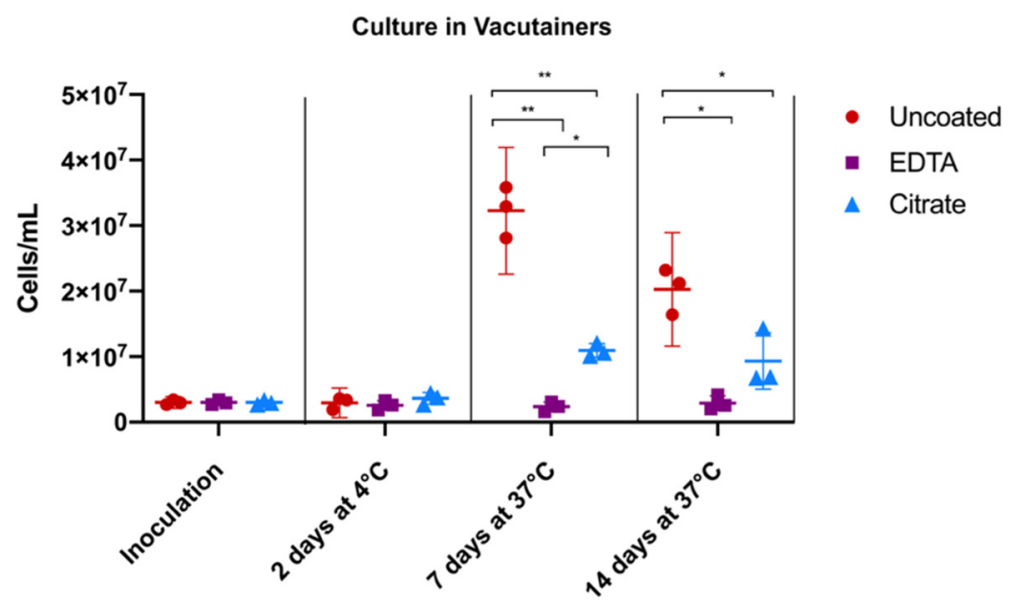

B

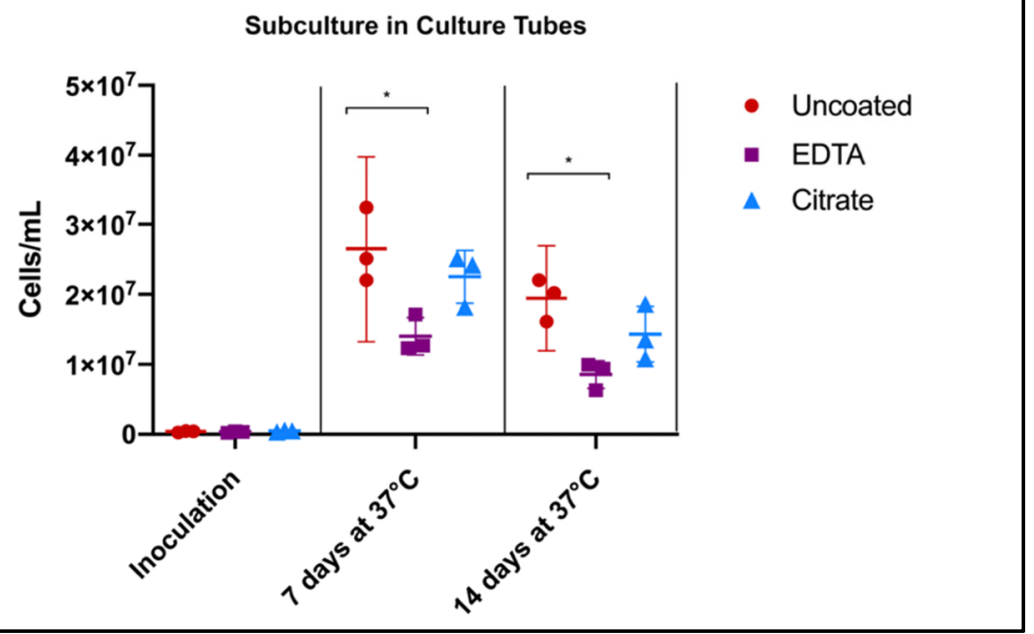

Figure 2. Influence of anticoagulants on Borrelia cultivability. To consider anticoagulant impact on Borrelia culture growth, we performed cell counts following inoculation of Borrelia into BSK broth and incubation in uncoated, EDTA, and citrate vacutainers, monitoring (A) the direct effects of anticoagulants on pathogen growth, and (B) consequences upon subcultivation. Culture was aliquoted into fresh BSK in an uncoated culture tube after $48 \mathrm{~h}$ in vacutainers (from A), to mimic standard shipping and laboratory culture procedure. Horizontal lines represent mean of three biological replicates and whiskers display the standard deviation. Asterisks indicate a significant difference $\left({ }^{*}=p<0.05\right.$, ** $=p<0.0001)$ by one-way ANOVA and Tukey's multiple comparisons post-hoc test $(n=3)$.

\subsection{Fraction Enrichment of Borrelia during Blood Processing}

Serum and plasma are most commonly used as the inoculation source for clinical culture, yet few studies have ventured into testing whole blood by molecular techniques. Our goal was therefore to evaluate whether the liquid component of blood is truly the best source of Borrelia, in order to identify the optimal blood fraction for direct detection purposes.

In this experiment, citrated whole blood (WB) collected from healthy volunteers was experimentally infected with Borrelia, incubated for thirty minutes, then centrifuged according to standard blood processing procedures to separate plasma from a cellular fraction consisting of erythrocytes, leukocytes and platelets. The results show that the liquid component of blood had far fewer Borrelia per field of view than the lower, cellular fraction $(p=0.0286)$ (Figure 3$)$. This finding indicates that valuable spirochetes are being separated into the host cell pellet rather than the plasma so often used for testing. Uninfected whole blood was included as a microscopy control. As an additional control, cleared 
plasma (PLS) was experimentally infected with Borrelia, and also showed more spirochetes per field of view in the lower fraction than the supernatant $(p=0.0318)$.

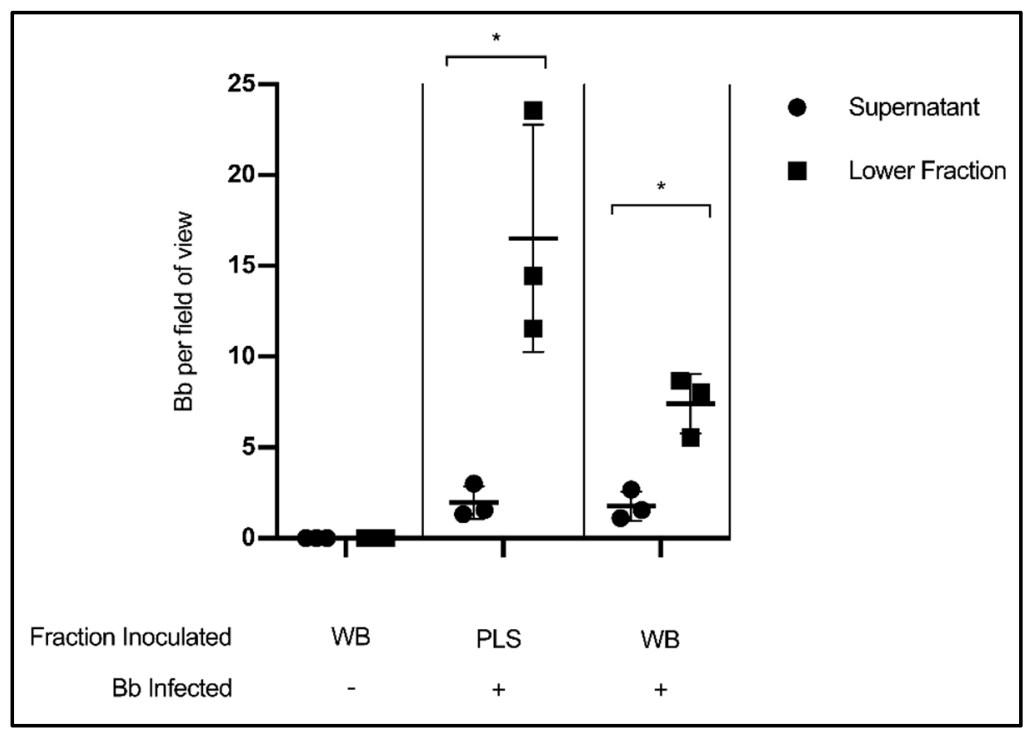

Figure 3. Stratification of Borrelia in blood following separation of cells and plasma. Quantification of Borrelia per field of view in the supernatant/liquid fraction and cellular/lower fraction following centrifugation at $400 \times g, 20 \mathrm{~min}$. Data presented as the means with standard deviation. Asterisks $\left.{ }^{*}=p<0.05\right)$ indicate significance difference using one-tailed paired t-tests $(n=3)$.

With the understanding that Borrelia partitions with host cells, it was of interest to pursue subsequent investigations of different blood fractions to identify where the greatest enrichment occurs. Whole blood was collected as above, and inoculated with $B$. Burgdorferi $(\mathrm{Bb})$ at a relatively conservative multiplicity of infection (MOI) of $1 \mathrm{Bb}: 18.37$ erythrocytes, in recognition of the low spirochetemic burden observed in disease. The blood was then separated into erythrocyte-leukocyte cellular fraction (ELCF), platelet-rich plasma (PRP), plasma (PLS) and platelet (PLT) fractions using the protocol depicted in Figure 4. Microscopy, culture, and molecular analyses were used to assess the fractions for Borrelia enrichment.

Immunofluorescent microscopy allowed for specific labelling of Borrelia (green) and platelets (red) (Figure 5A,B). The results of this investigation suggested that the platelet fraction consistently and drastically had the most Borrelia per field of view (Figure 5C). Co-localization between platelets and Borrelia, which we defined as direct juxtaposition of the two cell types, was observed in micrographs from both whole blood and platelet fractions. Enumeration of these co-localization events showed that $20.3 \%$ and $25.9 \%$ ( $p=0.338$, two-tailed $t$-test $)$ of platelets have Borrelia co-localized in whole blood and the platelet-enriched fraction, respectively (Table 1). Additionally, the spirochetes were noted to co-localize with erythrocytes in whole blood [51].

Table 1. Co-localization of Borrelia and platelets in experimentally infected whole blood and the platelet-enriched fraction obtained through centrifugation. Data collected as average counts per field of view with standard deviation from three biological replicates. Bb: B. burgdorferi; $\mathrm{Plt}=$ platelet.

\begin{tabular}{ccccc}
\hline Component & Avg Bb & Avg Plt & Bb-Plt/Total Bb & Plt-Bb/Total Plt \\
\hline Whole Blood & $22.8 \pm 4.1$ & $9.9 \pm 2.7$ & $8.9 \pm 2.0 \%$ & $20.3 \pm 5.4 \%$ \\
PLT Fraction & $53.9 \pm 4.7$ & $22.6 \pm 14.8$ & $11.3 \pm 5.6 \%$ & $25.9 \pm 7.1 \%$ \\
\hline
\end{tabular}




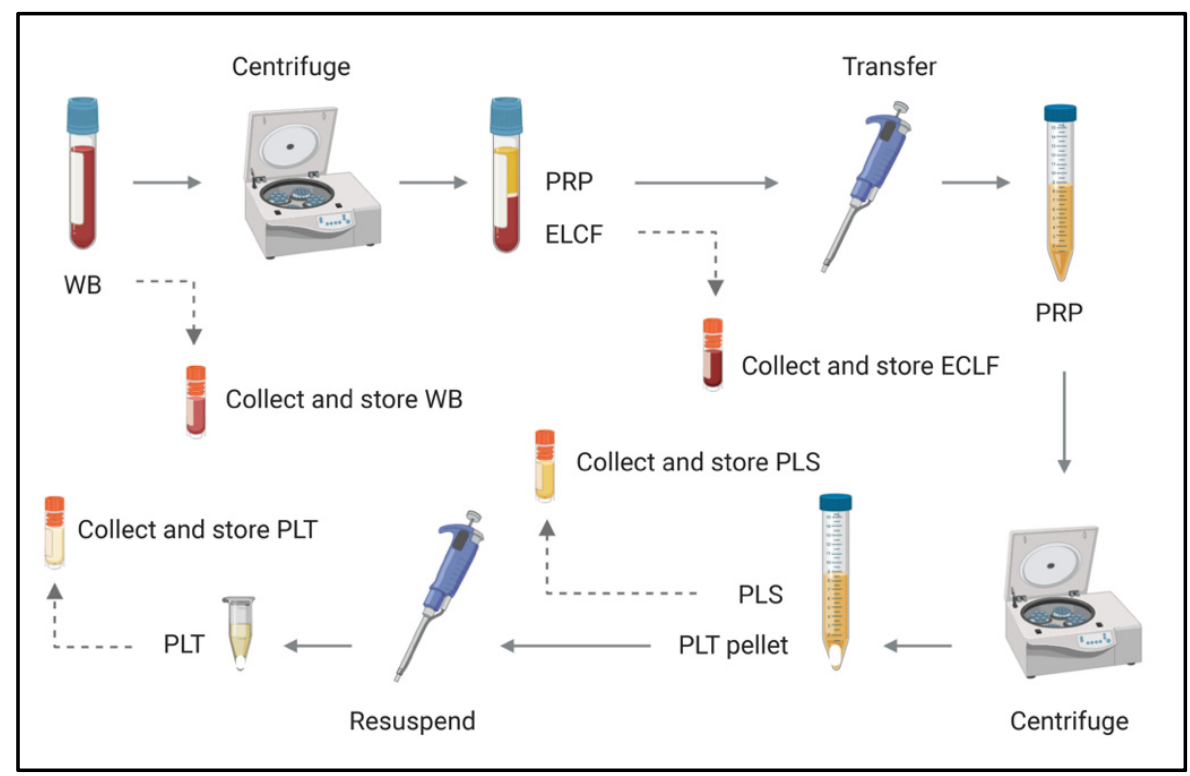

Figure 4. Schematic of blood fractionation protocol. Multiple steps were implemented to obtain a panel of blood components for comparison by microscopy, culture and molecular analyses. The initial centrifugation step pellets red and white blood cells at $120 \times g$ for $20 \mathrm{~min}$, leaving a platelet-rich plasma supernatant which is subsequently spun at $400 \times g$ for $20 \mathrm{~min}$ to obtain platelets (pellet) and acellular plasma (supernatant). WB = whole blood, ELCF = erythrocyte, leukocyte cellular fraction, $\mathrm{PRP}=$ platelet-rich plasma, $\mathrm{PLS}=$ plasma $($ platelet poor $)$, and PLT = platelet fraction .

The ability to culture from blood is an example of a relevant downstream application of optimized blood processing methods, and the accepted gold standard for detection of Borrelia infection. Based on the results of the previous experiment, the culture of Borrelia from the platelet fraction was compared to serum, a literature standard. Cultures were also inoculated using whole blood and ELCF; however, after one week, no growth could be visualized due to the highly heterogenous nature of the blood sample [51]. Additionally, PLS and PRP inoculated cultures showed no growth after 7 days and thus only serum was retained as the comparison to the prolific platelet pellet. As shown in Figure 6A, the platelet fraction displayed higher concentrations of spirochetes at both $7(p=0.0279)$ and 14 days $(p=0.0034)$ compared to the commonly used serum inoculum. Also of note, at 7 days, Borrelia were highly motile in the platelet culture, while the few cells visible in the serum culture were not, but by 14 days, there were motile spirochetes in both fractions.

An additional downstream application and method for comparing these blood fractions is through molecular analyses. Western blot analysis of protein content in each fraction using an OspA monoclonal antibody shows a strong band in the experimentally infected platelet fraction, comparable to the positive control of cultured Borrelia lysate (Figure 6(Bi)). Faint bands can be seen in the experimentally infected WB and ELCF and a very faint, barely visible band, can be seen in the infected plasma fraction. Diagnostic PCR specific for the $16 \mathrm{~S}$ rRNA gene of B. burgdorferi was attempted for each fraction without any DNA isolation, and the platelet fraction was the only sample, aside from the positive control lysate, to generate a visible band by agarose gel electrophoresis (Figure 6(Bii)). 


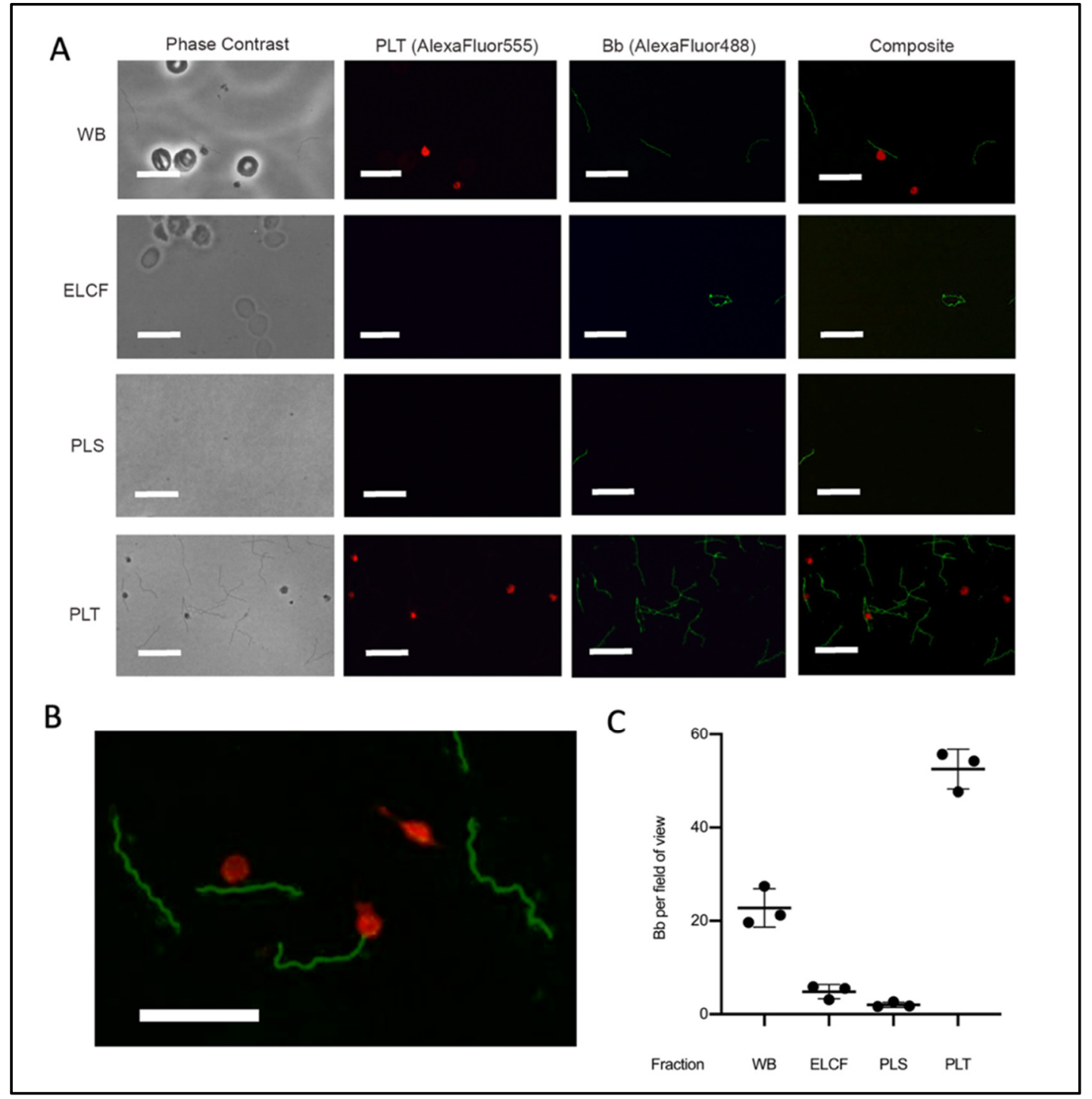

Figure 5. Microscopy of experimentally infected blood before and after fractionation. (A) Representative immunofluorescent micrographs of Borrelia (green) in fractions collected from experimentally infected whole blood (platelets labelled red). Scale bar $=13 \mu \mathrm{m}$. (B) Magnification of PLT fraction composite demonstrating co-localization. Scale bar $=13 \mu \mathrm{m}$. (C) Corresponding quantification of Borrelia per field of view from A; $n=3$. All comparisons by ANOVA and Tukey's multiple comparisons post-hoc test were significant with $p<0.005$ except red blood cells and plasma. Data represented as the means with standard deviation. WB = whole blood, ELCF = erythrocyte, leukocyte cellular fraction, PRP = platelet-rich plasma, PLS = plasma (platelet poor), and PLT = platelet fraction. 


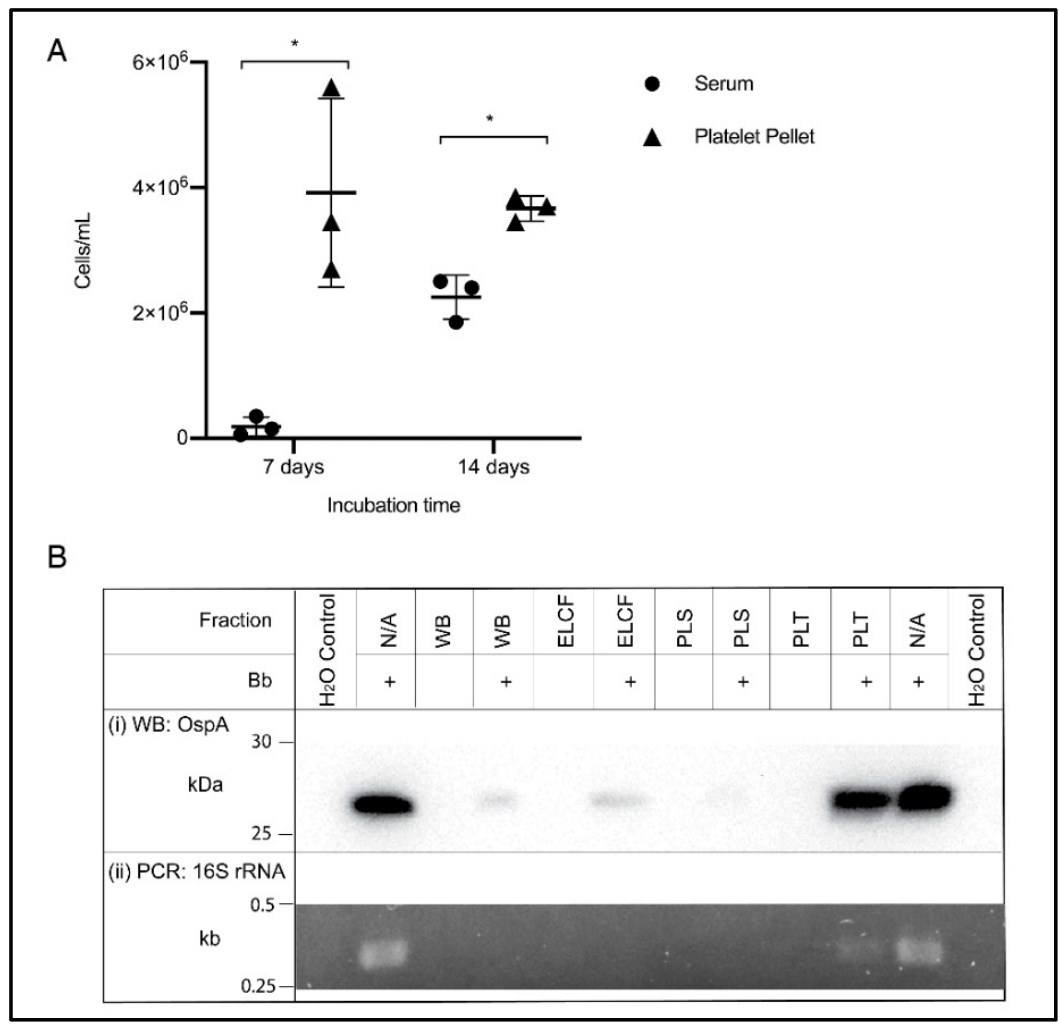

Figure 6. Culture and molecular detection of Borrelia in blood fractions. (A) Broth culture of serum and platelet fractions collected from experimentally infected blood samples, monitored weekly for growth. Data represented as the means with standard deviation. Asterisks indicate significant difference by one-tailed paired $t$-test $(*=p<0.05)(n=3)$. (B) Detection of OspA protein by Western blot (i) and the 16S rRNA gene by PCR (ii) in experimentally infected and matched uninfected blood, and controls. $\mathrm{WB}=$ whole blood, ELCF = erythrocyte, leukocyte cellular fraction, PLS = plasma, and PLT $=$ platelet fraction

\section{Discussion}

The experimentally infected human blood system evaluated in this study demonstrates that blood processing substantially influences the ability to recover and detect Borrelia. Methodological choices in the literature are often unfounded, and there is a lack of consensus between studies. Our findings establish that the frequently used anticoagulant (EDTA), and routine inoculum sources (plasma and serum), are not ideal for applications that directly detect Borrelia. As previously suggested, sodium citrate anticoagulant is superior to EDTA for the recovery of Borrelia, leading to a greater peak culture density. Routine fractionation of blood into plasma and a cell pellet segregates Borrelia into the cellular fraction, leaving the plasma with few spirochetes. A deeper analysis of multiple blood fractions using microscopy, culture, and molecular techniques identified that the platelet fraction obtained through centrifugation is highly concentrated with Borrelia, providing a novel reservoir of detectable Borrelia targets in the blood. Together, these findings identify opportunities for protocol optimization and provide refined guidelines for direct detection.

The risk of losing or altering Borrelia begins the moment blood is drawn into a vacutainer. Previous studies suggested that EDTA is inferior to citrate, potentially due to its stronger calcium and magnesium chelation properties [48]. One study of Borrelia miyamotoi, a species related to tick-borne relapsing fever pathogens, found the minimum inhibitory concentration of EDTA to be $0.25 \mathrm{mg} / \mathrm{mL}$, while the nominal concentration in an EDTA vacutainer is $1.8 \mathrm{mg} / \mathrm{mL}$ [52]. Our results provide additional evidence that EDTA inhibits Borrelia growth, while demonstrating the extent to which a clinically relevant workflow impacts cultivability. In clinical samples that already have a low concentration of spirochetes, 
reducing growth capacity by roughly half through EDTA exposure would severely decrease the ability to obtain positive culture results. Sodium citrate reached $85 \%$ of normal growth in our assessment, and therefore maximizes recovery from whole-blood components. These results are also relevant for molecular analyses of viable, minimally processed spirochetes, as appropriate handling ideally limits antigenic variation and metabolic shifts [53]. Ultimately, reducing procedural artifacts and preserving the integrity of the pathogen provides the greatest opportunity to obtain accurate direct test results.

Subsequent steps of direct testing from blood often involve separation of whole blood to obtain the desired blood component, typically plasma or serum. Our analysis of serum as an inoculum did not show appreciable growth until 14 days, indicating the starting concentration was either very low, or spirochetes were affected by the clotting process. It has been hypothesized that the coagulation process used to generate serum may trap spirochetes within the clot, Borrelia may bind to activated platelets, or substances could be released that adversely impact bacteria [34]. Inoculating with plasma avoids the negative impacts of clot formation, and reportedly yields more positive culture results compared to matched serum [34]. However, our results show that the vast majority of spirochetes are concentrated in the cellular component after processing experimentally infected whole blood to recover plasma, suggesting that plasma is also not an ideal inoculum source. It should be noted, however, that reports of plasma-based analysis often fail to distinguish between platelet-rich and cleared plasma. Based on our findings, we anticipate that this distinction could impact recovery considerably. Nevertheless, cellular fractions have rarely been investigated in the literature as Borrelia reservoirs. Our observations demonstrate that centrifugation as low as $400 \times g$ contributes to the concentration of spirochetes, which was a surprising result as $1000-8000 \times g$ forces are typically used to pellet Borrelia from in vitro culture [54-56]. Gravitational separation improved the yield of Borrelia DNA in one study [28], and may represent an alternative to centrifugation to limit Borrelia loss during liquid recovery. However, co-localization was observed between platelets and Borrelia (Table 1), as well as erythrocytes and Borrelia [51]. As a result of these interactions, a proportion of spirochetes may associate with platelets, erythrocytes, and even lymphocytes, separating into the cellular fraction regardless of centrifugation [57]. Yet, examination of individual fractions revealed that putative red blood cell interactions did not result in a robust pathogen yield according to cell counts (Figure 5) or molecular target detection (Figure 6B) in the erythrocyte and leukocyte cell pellet, which contains approximately 600 red blood cells for every 1 white blood cell in the average human. It was likewise not possible to obtain a quantifiable Borrelia culture from whole blood or the ELCF due to the abundance of host material. Therefore, any marginal improvement in Borrelia recovery achieved by generating a single heterogeneous cell pellet (as in Figure 3) is offset by the considerable dilution effects from the host cells. Meanwhile, the consistent platelet-Borrelia co-localization dynamics observed in whole blood and the separated platelet fraction support the hypothesis that Borrelia enrichment observed in the platelet fraction could be partly mediated by host cell associations. A few studies have previously considered Borrelia-platelet interactions, and concluded that Borrelia burgdorferi (sensu stricto), Borrelia garinii, and Borrelia afzellii as well as relapsing fever (RF)-causing Borrelia hermsii are capable of associating with activated platelets $[17,58,59]$. Host-pathogen interactions with various blood cells may help to identify where Borrelia are found within the blood.

Indeed, multiple modes of analysis, including immunofluorescent microscopy, culture and molecular detection, corroborate that the platelet fraction is enriched with Borrelia in our experimental infection model. This observation identifies for the first time that the platelets isolated by two-step centrifugation could provide a reservoir of detectable targets with immense opportunity for further exploration. Early studies in rats and humans briefly alluded to the use of platelet-rich plasma as a superior source of host-derived Borrelia $[17,22]$, although this observation was never investigated in more detail. The distribution of spirochetes between blood fractions has also been considered in the context of RF Borrelia and blood transfusions [60]. In this study, Thorp and Tonnetti found the majority of RF spirochetes within the red blood cell fraction; however, they diluted the platelet fraction 
substantially. Their findings nevertheless support the conclusion that the liquid fraction of blood is not an ideal source for direct testing.

The platelet fraction similarly displays promise as a culture inoculum, presenting with a highly motile culture at higher peak concentration than serum in a much shorter time span of only 7 days. For culture it is particularly important to obtain high concentrations of spirochetes as $5 \times 10^{4}$ cells $/ \mathrm{mL}$ is the lower limit for enumeration via Petroff-Hausser counting chamber. Despite these potential advances in growth rate, culture will likely still be too slow and potentially too insensitive for clinical utility but remains a valuable research tool. Comparatively, PCR can be a much more sensitive technique that can reportedly detect as low as 10-100 copy numbers and has been used in Lyme disease research for Borrelia detection in blood [61]. The majority of PCR-based studies test serum or plasma, but as the results of this study show, the enriched platelet fraction could further improve PCR detection. Of the available blood cell fractions, enrichment of Borrelia with platelets is particularly valuable in the diagnostic context because they have limited quantities of eukaryotic DNA, RNA [62], and inhibitory substances, which can dilute molecular pathogen targets and/or interfere with PCR amplification in the leukocyte and erythrocyte fraction [63]. Research on Borrelia protein content in blood is limited, but our analysis demonstrates that outer surface protein detection by Western blot is possible with the concentrated platelet fraction. Regardless of the target, the challenges facing direct detection may be ameliorated, at least in part, by probing the enriched fraction using various tools that have come to fruition across the Lyme disease literature.

Although the outcomes of our studies are clear under the conditions tested, Borrelia are diverse and complex bacteria, and their associations with the host are incompletely characterized. Parameters that could be varied to further contextualize these findings include the origin and derivation of Borrelia strains used (lab-adapted vs. clinically isolated), centrifugation speeds, infection time, platelet count, and bacterial MOI in blood. The highest MOI used in this study was 1 Bb:20 erythrocytes for experimental infection of whole blood, which is significantly lower than other host-cell interaction studies. However, this inoculation strategy resulted in a ratio of $2.3 \mathrm{Bb}: 1 \mathrm{Plt}$ in whole blood and in the platelet fraction after centrifugation. Studies investigating host-pathogen associations typically have more Borrelia present than host cells, spanning MOIs of 5 spirochetes: 1 host cell on the low end of the spectrum, up to 5000 Borrelia/host cell [64]. Increasing the pathogen burden and/or incubation length in the infection model could result in a number of physical and physiological changes that alter how Borrelia partition in the blood, potentially enhancing the phenomenon we describe here. Ultimately, evaluating the Borrelia content of platelets obtained from naturally infected LD patients would provide the most relevant insight into the clinical applicability of this blood fraction.

\section{Conclusions}

This research provides foundational work in the field of blood collection and processing for direct detection of Borrelia in blood. Questioning the seemingly benign methodological choices led to the discovery that the platelet fraction collected from citrated whole blood is a Borrelia-enriched reservoir. These findings should be used both to re-interpret past studies with suboptimal methodology, and to guide forthcoming research. Future studies should consider the implications of each methodological choice involved in blood collection, processing, and testing. The findings presented here advance our understanding of direct pathogen detection, and may contribute to faster, more reliable, and clinically useful diagnostic tools for Lyme disease.

Supplementary Materials: The following are available online at http://www.mdpi.com/2079-7737/9/11/366/s1, Figure S1: original western blot figures.

Author Contributions: V.P.S.: experimental design and execution, project administration, analysis, writing (original), and review/editing; I.L.M.: methodology (Borrelia culture), experimental guidance, and review/editing; L.V.: methodology (experimental infection assistance); C.M.K.: experimental guidance and review/editing; M.K.B.W.: conception, experimental design, funding acquisition, project administration, writing (original), and review/editing. All authors have read and agreed to the published version of the manuscript. 
Funding: This work was supported by research funds from the G. Magnotta Foundation for Vector-Borne Diseases to Melanie K. B. Wills, and a Natural Sciences and Engineering Research Council Discovery Grant (\#371639) to Cezar M. Khursigara.

Acknowledgments: The authors wish to thank Amy Tucker and Premila Sathasivam at the Human Nutraceutical Research Unit, University of Guelph, for their assistance with blood collection. We are also grateful to Rebecca Walchyshyn (Molecular and Cellular Biology) for her help optimizing PCR.

Conflicts of Interest: The authors declare no conflict of interest. The funders had no role in the design of the study; in the collection, analyses, or interpretation of data; in the writing of the manuscript, or in the decision to publish the results.

\section{Appendix A}

Table A1. Summary of methods used in Lyme disease clinical culture studies. Consideration was given to which blood components were used for inoculation of culture, what anticoagulant was present in collection tubes for whole blood and plasma collection and whether centrifugation steps were implemented for serum and plasma separation from whole blood. Data compiled by searching (("Borrelia burgdorferi") AND ("culture)) AND ("blood") with the requirement that blood-based clinical culture was conducted in the study and both inoculation source and collection tube were clearly indicated. Colour coding represents collection tube (red: uncoated, purple: EDTA, blue: citrate, and green: heparin) and studies were ordered chronologically to present vacutainer usage over time. Literature search was conducted on 15 January 2020, and updated on 23 June 2020 using PubMed.

\begin{tabular}{|c|c|c|c|}
\hline Inoculation Fraction & Collection Tube & Centrifugation & Citation \\
\hline Plasma & EDTA & Unspecified & Horn et al. 2020 [40] \\
\hline Serum (+ a few blood cells) & Uncoated & Yes (low speeds) & Middelveen et al. 2018 [9] \\
\hline Serum (+ a few blood cells) & Uncoated & Yes (low speeds) & Middelveen et al. 2015 [41] \\
\hline Serum and whole blood & $\begin{array}{l}\text { Uncoated, EDTA and } \\
\text { direct into BSK }\end{array}$ & No & Sapi et al. 2013 [42] \\
\hline Plasma & EDTA & Yes $(260 \times g, 15 \mathrm{~min})$ & Liveris et al. 2012 [43] \\
\hline Plasma & EDTA & Yes $(260 \times g, 15 \mathrm{~min})$ & Liveris et al. 2011 [44] \\
\hline Plasma & Citrate & Yes (800 rpm, $10 \mathrm{~min})$ & Maraspin et al. 2011 [45] \\
\hline Plasma & Citrate & Yes $(100 \times g, 5 \mathrm{~min})$ & Cerar et al., 2008 [46] \\
\hline Plasma & EDTA & Yes $(350 \times g, 15 \mathrm{~min})$ & Coulter et al. 2005 [29] \\
\hline Plasma & EDTA & Unspecified & Wormser et al. 2005 [30] \\
\hline Plasma & EDTA & Yes $(260 \times g, 15 \mathrm{~min})$ & Wormser et al. 2001 [31] \\
\hline Plasma & Citrate & Yes (800 rpm, $5 \mathrm{~min})$ & Arnez et al. 2001 [32] \\
\hline Plasma & EDTA & Unspecified & Marques et al. 2000 [33] \\
\hline Serum and plasma & Uncoated and EDTA & Yes $(260 \times g, 15 \mathrm{~min})$ & Wormser et al. 2000 [34] \\
\hline $\begin{array}{c}\text { Serum, plasma and whole } \\
\text { blood }\end{array}$ & Uncoated and heparin & Unspecified & Liveris et al. 1999 [35] \\
\hline Whole blood & EDTA & No & Phillips et al. 1998 [36] \\
\hline Serum and whole blood & Heparin & $\begin{array}{l}\text { Yes (serum-1100×g, } \\
10 \mathrm{~min})\end{array}$ & Wormser et al. 1998 [37] \\
\hline $\begin{array}{l}\text { Serum, whole blood, plasma, } \\
\text { buffy coat and erythrocytes }\end{array}$ & Uncoated and EDTA & Yes $(260 \times g, 15 \mathrm{~min})$ & Goodman et al. 1995 [22] \\
\hline Whole blood & $\begin{array}{l}\text { Uncoated (directly into } \\
\text { BSK) }\end{array}$ & No & Berger et al. 1994 [38] \\
\hline Serum and whole blood & Uncoated and heparin & No & Wallach et al. 1993 [39] \\
\hline Serum and whole blood & Uncoated and heparin & $\begin{array}{l}\text { Yes (serum-500× } g, \\
10 \mathrm{~min})\end{array}$ & Nadelman 1990 [14] \\
\hline
\end{tabular}




\section{References}

1. Nelson, C.A.; Saha, S.; Kugeler, K.J.; Delorey, M.J.; Shankar, M.B.; Hinckley, A.F.; Mead, P.S. Incidence of Clinician-Diagnosed Lyme Disease, United States, 2005-2010. Emerg. Infect. Dis. 2015, 21, 1625-1631.

2. Schutzer, S.E.; Body, B.A.; Boyle, J.; Branson, B.M.; Dattwyler, R.J.; Fikrig, E.; Gerald, N.J.; Gomes-Solecki, M.; Kintrup, M.; Ledizet, M.; et al. Direct Diagnostic Tests for Lyme Disease. Clin. Infect. Dis. 2019, 68, 1052-1057.

3. Feder, H.M., Jr.; Abeles, M.; Bernstein, M.; Whitaker-Worth, D.; Grant-Kels, J.M. Diagnosis, treatment, and prognosis of erythema migrans and Lyme arthritis. Clin. Dermatol. 2006, 24, 509-520. [CrossRef]

4. Godar, D.A.; Laniosz, V.; Wetter, D.A. Lyme Disease Update for the General Dermatologist. Am. J. Clin. Dermatol. 2015, 16, 5-18.

5. Fix, A.D.; Peña, C.A.; Strickland, G.T. Racial differences in reported lyme disease incidence. Am. J. Epidemiol. 2000, 152, 756-759. [CrossRef]

6. Goddard, J. Not All Erythema Migrans Lesions Are Lyme Disease. Am. J. Med. 2017, 130, 231-233. [CrossRef]

7. Kannangara, D.W.; Patel, P. Report of non-lyme, erythema migrans rashes from New Jersey with a review of possible role of tick salivary toxins. Vector Borne Zoonotic Dis. 2018, 18, 641-652. [CrossRef]

8. Aucott, J.N.; Rebman, A.W.; Crowder, L.A.; Kortte, K.B. Post-treatment Lyme disease syndrome symptomatology and the impact on life functioning: Is there something here? Qual. Life Res. 2013, $22,75-84$.

9. Middelveen, M.; Sapi, E.; Burke, J.; Filush, K.; Franco, A.; Fesler, M.; Stricker, R. Persistent Borrelia Infection in Patients with Ongoing Symptoms of Lyme Disease. Healthcare 2018, 6, 33-51.

10. Bamm, V.V.; Ko, J.T.; Mainprize, I.L.; Sanderson, V.P.; Wills, M.K.B. Lyme Disease Frontiers: Reconciling Borrelia Biology and Clinical Conundrums. Pathogens 2019, 8, 299.

11. Branda, J.A.; Body, B.A.; Boyle, J.; Branson, B.M.; Dattwyler, R.J.; Fikrig, E.; Gerald, N.J.; Gomes-Solecki, M.; Kintrup, M.; Ledizet, M.; et al. Advances in Serodiagnostic Testing for Lyme Disease Are at Hand. Clin. Infect. Dis. 2017, 66, 1133-1139. [CrossRef]

12. Rebman, A.W.; Crowder, L.A.; Kirkpatrick, A.; Aucott, J.N. Characteristics of seroconversion and implications for diagnosis of post-treatment Lyme disease syndrome: Acute and convalescent serology among a prospective cohort of early Lyme disease patients. Clin. Rheumatol. 2014, 34, 585-589. [CrossRef]

13. Fallon, B.A.; Pavlicova, M.; Coffino, S.W.; Brenner, C. A Comparison of Lyme Disease Serologic Test Results From 4 Laboratories in Patients with Persistent Symptoms After Antibiotic Treatment. Clin. Infect. Dis. 2014, 59, 1705-1710. [CrossRef] [PubMed]

14. Nadelman, R.B.; Pavia, C.S.; Magnarelli, L.A.; Wormser, G.P. Isolation of Borrelia burgdorferi from the blood of seven patients with lyme disease. Am. J. Med. 1990, 88, 21-26. [CrossRef]

15. Goodman, J.L.; Jurkovich, P.; Kramber, J.M.; Johnson, R.C. Molecular detection of persistent Borrelia burgdorferi in the urine of patients with active Lyme disease. Infect. Immun. 1991, 59, 269-278. [CrossRef]

16. Dorward, D.W.; Schwan, T.G.; Garon, C.F. Immune capture and detection of Borrelia burgdorferi antigens in urine, blood, or tissues from infected ticks, mice, dogs, and humans. J. Clin. Microbiol. 1991, 29, 1162-1170. [CrossRef]

17. Galbe, J.L.; Guy, E.; Zapatero, J.M.; Peerschke, E.I.B.; Benach, J.L. Vascular clearance of Borrelia burgdorferi in rats. Microb. Pathog. 1993, 14, 187-201. [CrossRef]

18. Pritt, B.S.; Mead, P.S.; Johnson, D.K.H.; Neitzel, D.F.; Respicio-Kingry, L.B.; Davis, J.P.; Schiffman, E.; Sloan, L.M.; Schriefer, M.E.; Replogle, A.J.; et al. Identification of a novel pathogenic Borrelia species causing Lyme borreliosis with unusually high spirochaetaemia: A descriptive study. Lancet Infect. Dis. 2016, 16, 556-564. [CrossRef]

19. Waddell, L.A.; Greig, J.; Mascarenhas, M.; Harding, S.; Lindsay, R.; Ogden, N. The Accuracy of Diagnostic Tests for Lyme Disease in Humans, A Systematic Review and Meta-Analysis of North American Research. PLoS ONE 2016, 11, e0168613. [CrossRef] [PubMed]

20. Wormser, G.P.; Brisson, D.; Liveris, D.; Hanincova, K.; Sandigursky, S.; Nowakowski, J.; Nadelman, R.B.; Ludin, S.; Schwartz, I. Borrelia burgdorferi genotype predicts the capacity for hematogenous dissemination during early Lyme disease. J. Infect. Dis. 2008, 198, 1358-1364. [CrossRef]

21. Schmidt, B.L. PCR in laboratory diagnosis of human Borrelia burgdorferi infections. Clin. Microbiol. Rev. 1997, 10, 185-201. [CrossRef] 
22. Goodman, J.L.; Bradley, J.F.; Ross, A.E.; Goellner, P.; Lagus, A.; Vitale, B.; Berger, B.W.; Luger, S.; Johnson, R.C. Bloodstream invasion in early Lyme disease: Results from a prospective, controlled, blinded study using the polymerase chain reaction. Am. J. Med. 1995, 99, 6-12. [CrossRef]

23. Liveris, D.; Schwartz, I.; McKenna, D.; Nowakowski, J.; Nadelman, R.B.; DeMarco, J.; Iyer, R.; Cox, M.E.; Holmgren, D.; Wormser, G.P. Quantitation of cell-associated borrelial DNA in the blood of Lyme disease patients with erythema migrans. Eur. J. Clin. Microbiol. Infect. Dis. 2012, 31, 791-795. [CrossRef] [PubMed]

24. Wormser, G.P. Hematogenous dissemination in early Lyme disease. Wien. Klin. Wochenschr. 2006, 118, 634-637. [CrossRef] [PubMed]

25. Mosel, M.R.; Carolan, H.E.; Rebman, A.W.; Castro, S.; Massire, C.; Ecker, D.J.; Soloski, M.J.; Aucott, J.N.; Eshoo, M.W. Molecular Testing of Serial Blood Specimens from Patients with Early Lyme Disease during Treatment Reveals Changing Coinfection with Mixtures of Borrelia burgdorferi Genotypes. Antimicrob. Agents Chemother. 2019, 63, 1625-1626.

26. Branda, J.A.; Lemieux, J.E.; Blair, L.; Ahmed, A.A.; Hong, D.K.; Bercovici, S.; Blauwkamp, T.A.; Hollemon, D.; Ho, C.; Strle, K.; et al. Detection of Borrelia burgdorferi Cell-free DNA in Human Plasma Samples for Improved Diagnosis of Early Lyme Borreliosis. Clin. Infect. Dis. 2020. [CrossRef]

27. Nilsson, R.J.A.; Balaj, L.; Hulleman, E.; van Rijn, S.; Pegtel, D.M.; Walraven, M.; Widmark, A.; Gerritsen, W.R.; Verheul, H.M.; Vandertop, W.P.; et al. Blood platelets contain tumor-derived RNA biomarkers. Blood 2011, 118, 3680-3683. [CrossRef]

28. Lee, S.H.; Vigliotti, V.S.; Vigliotti, J.S.; Jones, W.; Pappu, S. Increased Sensitivity and Specificity of Borrelia burgdorferi 16S Ribosomal DNA Detection. Am. J. Clin. Pathol. 2010, 133, 569-576. [CrossRef]

29. Coulter, P.; Lema, C.; Flayhart, D.; Linhardt, A.S.; Aucott, J.N.; Auwaerter, P.G.; Dumler, J.S. Two-Year Evaluation of Borrelia burgdorferi Culture and Supplemental Tests for Definitive Diagnosis of Lyme Disease. J. Clin. Microbiol. 2005, 43, 5080-5084. [CrossRef]

30. Wormser, G.P.; McKenna, D.; Carlin, J.; Nadelman, R.B.; Cavaliere, L.F.; Holmgren, D.; Byrne, D.W.; Nowakowski, J. Brief Communication: Hematogenous Dissemination in Early Lyme Disease. Ann. Intern. Med. 2005, 142, 751. [CrossRef]

31. Wormser, G.P.; Bittker, S.; Cooper, D.; Nowakowski, J.; Nadelman, R.B.; Pavia, C. Yield of Large-Volume Blood Cultures in Patients with Early Lyme Disease. J. Infect. Dis. 2001, 184, 1070-1072. [CrossRef] [PubMed]

32. Arnež, M.; Ružić-Sabljić, E.; Ahčan, J.; Radšel-Medvešček, A.; Pleterski-Rigler, D.; Strle, F. Isolation of Borrelia burgdorferi sensu lato from blood of children with solitary erythema migrans. Pediatr. Infect. Dis. J. 2001, 20, 251-255. [CrossRef] [PubMed]

33. Marques, A.R.; Stock, F.; Gill, V. Evaluation of a new culture medium for Borrelia burgdorferi. J. Clin. Microbiol. 2000, 38, 4239-4241. [CrossRef]

34. Wormser, G.P.; Bittker, S.; Cooper, D.; Nowakowski, J.; Nadelman, R.B.; Pavia, C. Comparison of the yields of blood cultures using serum or plasma from patients with early Lyme disease. J. Clin. Microbiol. 2000, 38, 1648-1650. [CrossRef] [PubMed]

35. Liveris, D.; Varde, S.; Iyer, R.; Koenig, S.; Bittker, S.; Cooper, D.; McKenna, D.; Nowakowski, J.; Nadelman, R.B.; Wormser, G.P.; et al. Genetic diversity of Borrelia burgdorferi in lyme disease patients as determined by culture versus direct PCR with clinical specimens. J. Clin. Microbiol. 1999, 37, 565-569. [CrossRef] [PubMed]

36. Phillips, S.E.; Mattman, L.H.; Hulínská, D.; Moayad, H. A proposal for the reliable culture of Borrelia burgdorferi from patients with chronic lyme disease, even from those previously aggressively treated. Infection 1998, 26, 364-367.

37. Wormser, G.P.; Nowakowski, J.; Nadelman, R.B.; Bittker, S.; Cooper, D.; Pavia, C. Improving the yield of blood cultures for patients with early lyme disease. J. Clin. Microbiol. 1998, 36, 296-298.

38. Berger, B.W.; Johnson, R.C.; Kodner, C.; Coleman, L. Cultivation of Borrelia burgdorferi from the blood of two patients with erythema migrans lesions lacking extracutaneous signs and symptoms of Lyme disease. J. Am. Acad. Dermatol. 1994, 30, 48-51.

39. Wallach, F.R.; Forni, A.L.; Hariprashad, J.; Stoeckle, M.Y.; Steinberg, C.R.; Fisher, L.; Malawista, S.E.; Murray, H.W. Circulating Borrelia burgdotferi in Patients with Acute Lyme Disease: Results of Blood Cultures and Serum DNA Analysis. J. Infect. Dis. 1993, 168, 1541-1543. 
40. Horn, E.J.; Dempsey, G.; Schotthoefer, A.M.; Prisco, U.L.; McArdle, M.; Gervasi, S.S.; Golightly, M.; De Luca, C.; Evans, M.; Pritt, B.S.; et al. The Lyme Disease Biobank - Characterization of 550 Patient and Control Samples from the East Coast and Upper Midwest of the United States. J. Clin. Microbiol. 2020, 58, 1-12. [CrossRef]

41. Middelveen, M.J.; Bandoski, C.; Burke, J.; Sapi, E.; Filush, K.R.; Wang, Y.; Franco, A.; Mayne, P.J.; Stricker, R.B. Exploring the association between Morgellons disease and Lyme disease: Identification of Borrelia burgdorferi in Morgellons disease patients. BMC Dermatol. 2015, 15, 1-14. [CrossRef]

42. Sapi, E.; Pabbati, N.; Datar, A.; Davies, E.M.; Rattelle, A.; Kuo, B.A. Improved culture conditions for the growth and detection of Borrelia from human serum. Int. J. Med. Sci. 2013, 10, 362-376. [CrossRef]

43. Liveris, D.; Schwartz, I.; McKenna, D.; Nowakowski, J.; Nadelman, R.; DeMarco, J.; Iyer, R.; Bittker, S.; Cooper, D.; Holmgren, D.; et al. Comparison of five diagnostic modalities for direct detection of Borrelia burgdorferi in patients with early Lyme disease. Diagn. Microbiol. Infect. Dis. 2012, 73, 243-245. [CrossRef]

44. Liveris, D.; Schwartz, I.; Bittker, S.; Cooper, D.; Iyer, R.; Cox, M.E.; Wormser, G.P. Improving the yield of blood cultures from patients with early lyme disease. J. Clin. Microbiol. 2011, 49, 2166-2168. [CrossRef] [PubMed]

45. Maraspin, V.; Ogrinc, K.; Ružić-Sabljić, E.; Lotrič-Furlan, S.; Strle, F. Isolation of Borrelia burgdorferi sensu lato from blood of adult patients with borrelial lymphocytoma, Lyme neuroborreliosis, Lyme arthritis and acrodermatitis chronica atrophicans. Infection 2011, 39, 35-40. [CrossRef]

46. Cerar, T.; Ogrinc, K.; Cimperman, J.; Lotrič-Furlan, S.; Strle, F.; Ružić-Sabljić, E. Validation of cultivation and PCR methods for diagnosis of Lyme neuroborreliosis. J. Clin. Microbiol. 2008, 46, 3375-3379. [CrossRef] [PubMed]

47. Rudenko, N.; Golovchenko, M.; Vancova, M.; Clark, K.; Grubhoffer, L.; Oliver, J.H., Jr. Isolation of live Borrelia burgdorferi sensu lato spirochaetes from patients with undefined disorders and symptoms not typical for Lyme borreliosis. Clin. Microbiol. Infect. 2016, 22, 267.e9-267.e15. [CrossRef] [PubMed]

48. Marques, A.R.; Yang, X.; Smith, A.A.; Zhuang, X.; Turk, S.; Williams, C.D.; Law, M.A.; Barbour, A.G. Citrate Anticoagulant Improves the burgdorferi Plasma Culture. J. Clin. Microbiol. 2017, 55, 3297-3299. [CrossRef] [PubMed]

49. Lin, Y.P.; Li, L.; Zhang, F.; Linhardt, R.J. Borrelia burgdorferi glycosaminoglycan-binding proteins: A potential target for new therapeutics against Lyme disease. Microbiology 2017, 163, 1759-1766. [CrossRef]

50. Nolte, O. Nucleic Acid Amplification Based Diagnostic of Lyme (Neuro-)borreliosis—Lost in the Jungle of Methods, Targets, and Assays? Open Neurol. J. 2012, 6, 129-139. [CrossRef]

51. Sanderson, V. University of Guelph, Guelph, ON, Canada. Unpublished work. 2020.

52. Koetsveld, J.; Kolyasnikova, N.M.; Wagemakers, A.; Toporkova, M.G.; Sarksyan, D.S.; Oei, A.; Platonov, A.E.; Hovius, J.W. Development and optimization of an in vitro cultivation protocol allows for isolation of Borrelia miyamotoi from patients with hard tick-borne relapsing fever. Clin. Microbiol. Infect. 2017, 23, 480-484. [CrossRef]

53. Singh, S.K.; Girschick, H.J. Molecular survival strategies of the Lyme disease spirochete. Infect. Dis. 2004, 4, 575-583.

54. Kersten, A.; Poitschek, C.; Rauch, S.; Aberer, E. Effects of penicillin, ceftriaxone, and doxycycline on morphology of Borrelia burgdorferi. Antimicrob. Agents Chemother. 1995, 39, 1127-1133. [CrossRef]

55. Murgia, R.; Cinco, M. Induction of cystic forms by different stress conditions in Borrelia burgdorferi. APMIS 2004, 112, 57-62. [CrossRef] [PubMed]

56. Motaleb, M.A.; Corum, L.; Bono, J.L.; Elias, A.F.; Rosa, P.; Samuels, D.S.; Charon, N.W. Borrelia burgdorferi periplasmic flagella have both skeletal and motility functions. Proc. Natl. Acad. Sci. USA 2000, 97, 10899-10904. [CrossRef]

57. Dorward, D.W.; Fischer, E.R.; Brooks, D.M. Invasion and Cytopathic Killing of Human Lymphocytes by Spirochetes Causing Lyme Disease. Clin. Infect. Dis. 1997, 25, S2-S8. [CrossRef]

58. Coburn, J.; Barthold, S.W.; Leong, J.M. Diverse Lyme disease spirochetes bind integrin alpha IIb beta 3 on human platelets. Infect. Immun. 1994, 62, 5559-5567. [CrossRef] [PubMed]

59. Coburn, J.; Leong, J.M.; Erban, J.K. Integrin alpha Illb beta 3 mediates binding of the Lyme disease agent Borrelia burgdorferi to human platelets. Proc. Natl. Acad. Sci. USA 1993, 90, 7059-7063. [CrossRef]

60. Thorp, A.M.; Tonnetti, L. Distribution and survival of Borrelia miyamotoi in human blood components. Transfusion 2016, 56, 705-711. [CrossRef] 
61. Eshoo, M.W.; Crowder, C.C.; Rebman, A.W.; Rounds, M.A.; Matthews, H.E.; Picuri, J.M.; Soloski, M.J.; Ecker, D.J.; Schutzer, S.E.; Aucott, J.N. Direct molecular detection and genotyping of Borrelia burgdorferi from whole blood of patients with early Lyme disease. PLOS ONE 2012, 7, 3-8. [CrossRef]

62. Bugert, P. (Ed.) DNA and RNA Profiling in Human Blood; Methods in Molecular Biology; Humana Press: Totowa, NJ, USA, 2009; Volume 496, ISBN 978-1-934115-93-0.

63. Al-Soud, W.A.; Radstrom, P. Purification and Characterization of PCR-Inhibitory Components in Blood Cells. J. Clin. Microbiol. 2001, 39, 485-493. [CrossRef] [PubMed]

64. Wu, J.; Weening, E.H.; Faske, J.B.; Höök, M.; Skare, J.T. Invasion of eukaryotic cells by Borrelia burgdorferi requires $\beta 1$ integrins and Src kinase activity. Infect. Immun. 2011, 79, 1338. [CrossRef]

Publisher's Note: MDPI stays neutral with regard to jurisdictional claims in published maps and institutional affiliations.

(C) 2020 by the authors. Licensee MDPI, Basel, Switzerland. This article is an open access article distributed under the terms and conditions of the Creative Commons Attribution (CC BY) license (http://creativecommons.org/licenses/by/4.0/). 